\title{
Quantum phases on demand
}

\author{
The need to control and manipulate the properties of quantum materials has led to a burst of interest in \\ their properties out of equilibrium. Understanding this regime represents an opportunity for theorists and \\ experimentalists alike.
}

$\mathrm{T}$ he rise of quantum materials has latterly been one of the more recognizable trends in physics. Since the term started to gain currency a few years $\mathrm{ago}^{2}$, we have witnessed an explosion of discoveries of topological phases of matter'; we've seen a burst of activity on twisted bilayer graphene, following the discovery that it can host unconventional superconductivity ${ }^{4}$; and we've learned a great deal about the counterintuitive phenomenon of many-body localization, which gives rise to entirely new phases of quantum matter when it is driven away from equilibrium ${ }^{5}$.

Experimentally, there is a range of methods available to push materials out of equilibrium, including switching on electric currents, the application of heat or laser pulses, and embedding them in quantum cavities. And as these approaches have improved in precision, it has become possible to bring about properties such as magnetism or superconductivity in systems that wouldn't otherwise display them.

Although formidable challenges remain before these phenomena can be harnessed for use in practical applications and devices, the vision of inducing properties in materials 'on demand' ${ }^{6}$ is inspiring researchers to work along new lines of enquiry. Some of these were on display at a recent symposium hosted by Columbia University and the Flatiron Institute in New York, held in order to mark the launch of the Max Planck-New York Center for Nonequilibrium Quantum Phenomena.

For example, as they report in a Letter in this issue, James McIver and colleagues have been able to induce the anomalous Hall effect in a sheet of graphene using a femtosecond pulse of circularly polarized light. Just as a superlattice structure can be used as a means to tailor a system's band structure, a periodic external drive can also be used to alter the effective band structure that governs the stroboscopic dynamics of electrons, known as Floquet-Bloch bands (depicted by an artist's impression in the figure). By detecting a Hall current without the application of a magnetic field, McIver

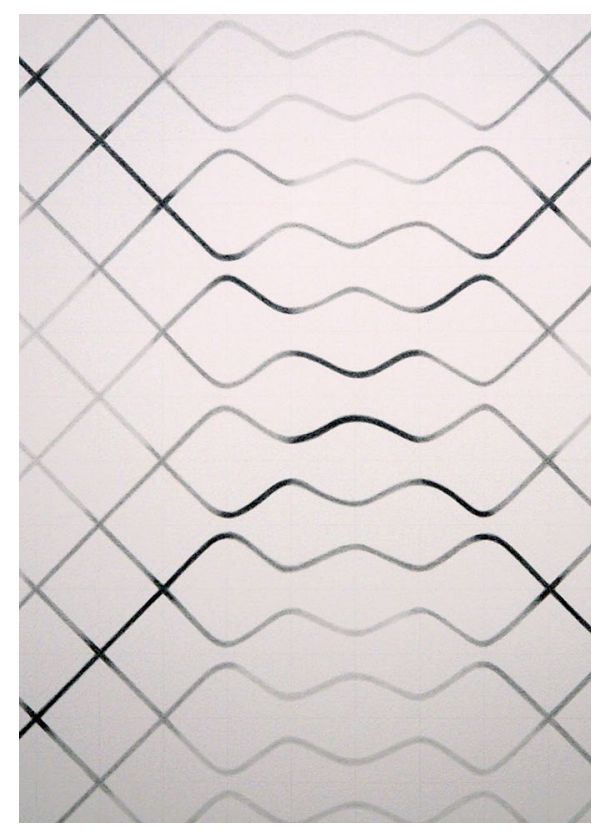

Credit: Robert Scott Whipkey

and colleagues show that it is possible to use this approach to transiently induce a topological phase in graphene.

As Justin Song describes in his News \& Views article introducing the experiment, this may come to represent a coming of age for the so-called Floquet engineering of quantum materials: the anomalous Hall effect arises from a Berry curvature that is coherently induced by light, in a material where none is present in equilibrium. The ability to modify the intrinsic properties of the electron wave function of a material in this way clearly opens up a wealth of tantalizing prospects: is it possible to induce dissipationless transport in devices on demand, for example? Can other properties, such as magnetism, be induced and controlled in this way as well? Such questions are now being actively pursued by a number of research groups around the world.

It is also worth noting the important role played by theory and computation in these efforts. The suggestion that Floquetengineered topological bands could be accessed in graphene (and indeed in other systems) came a decade $\mathrm{ago}^{7,8}$, for example. But more broadly, there is now a rich literature that has laid down the foundations for our understanding of quantum many-body systems driven out of equilibrium ${ }^{5}$. These have come along with a flurry of testable predictions, offering experimentalists an ever-growing list of questions to address and pursue.

In turn, the technical challenges that must be overcome in order to access the non-equilibrium regime empirically inspire the entire field to break new ground: again, returning to Floquet topological states as an example, the first experiments validating aspects of their theoretical proposal were carried out in synthetic physical settings such as photonic crystals ${ }^{9}$ and optically trapped ultracold atoms ${ }^{10}$. Moreover, the optical pulse techniques used by McIver and colleagues have been developed over many years as a means to coherently manipulate many different electronic systems away from equilibrium ${ }^{11}$.

The study of quantum materials out of equilibrium is therefore characterized by a close and fruitful interaction between theorists and experimentalists of different hues. As all the talks presented at the symposium in New York made clear, the tools for tailoring the properties of these systems on demand are now coming into place. Many interesting discoveries lie ahead.

Published online: 8 January 2020 https://doi.org/10.1038/s41567-019-0781-4

References

1. Nat. Phys. 12, 105 (2016)

. Keimer, B. \& Moore, J. E. Nat. Phys. 13, 1045-1055 (2017).

3. Wen, X.-G. Science 363, eaal3099 (2019).

4. Cao, Y. et al. Nature 556, 43-50 (2018).

5. Moessner, R. \& Sondhi, S. L. Nat. Phys. 13, 424-428 (2017).

6. Basov, D. N., Averitt, R. D. \& Hsieh, D. Nat. Mater. 16, 1077-1088 (2017).

7. Oka, T. \& Aoki, H. Phys. Rev. B 79, 081406(R) (2009).

8. Kitakawa, T. et al. Phys. Rev. B 84, 235108 (2011).

9. Rechtsman, M. C. et al. Nature 496, 196-200 (2013).

10. Jotzu, G. et al. Nature 515, 237-240 (2014).

11. Buzzi, M. et al. Nat. Rev. Mater. 3, 299-311 (2018). 